\title{
Virulence factors of Actinobacillus pleuropneumoniae involved in colonization, persistence and induction of lesions in its porcine host
}

\author{
Koen Chiers ${ }^{1 *}$, Tine De Waele ${ }^{1,2}$, Frank Pasmans ${ }^{1}$, Richard Ducatelle ${ }^{1}$, \\ Freddy HAESEBROUCK ${ }^{1}$ \\ ${ }^{1}$ Department of Pathology, Bacteriology and Poultry Diseases, Faculty of Veterinary Medicine, \\ Ghent University, Ghent, Belgium \\ ${ }^{2}$ Department of Virology, Parasitology and Immunology, Faculty of Veterinary Medicine, \\ Ghent University, Ghent, Belgium
}

(Received 28 November 2009; accepted 10 June 2010)

\begin{abstract}
Actinobacillus pleuropneumoniae is the causative agent of porcine pleuropneumonia. The virulence factors of this microorganism involved in colonization and the induction of lung lesions have been thoroughly studied and some have been well characterized. A. pleuropneumoniae binds preferentially to cells of the lower respiratory tract in a process involving different adhesins and probably biofilm formation. Apx toxins and lipopolysaccharides exert pathogenic effects on several host cells, resulting in typical lung lesions. Lysis of host cells is essential for the bacterium to obtain nutrients from the environment and A. pleuropneumoniae has developed several uptake mechanisms for these nutrients. In addition to persistence in lung lesions, colonization of the upper respiratory tract - and of the tonsils in particular - may also be important for long-term persistent asymptomatic infection. Information on virulence factors involved in tonsillar and nasal cavity colonization and persistence is scarce, but it can be speculated that similar features as demonstrated for the lung may play a role.
\end{abstract}

Actinobacillus pleuropneumoniae / virulence factor / pig / respiratory disease

\section{Table of contents}

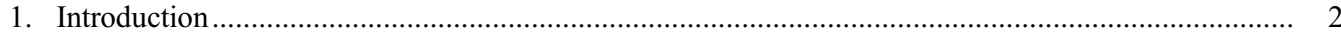

2. Interactions of $A$. pleuropneumoniae with the lower respiratory tract............................................. 2

2.1. Virulence factors involved in adhesion ............................................................................... 2

2.2. Virulence factors involved in the acquisition of essential nutrients ........................................... 4

2.3. Virulence factors involved in the induction of lesions ......................................................... 6

2.4. Virulence factors involved in avoiding the host's defense mechanisms .................................. 7

2.5. Virulence factors involved in persistence........................................................................ 8

3. Interactions of $A$. pleuropneumoniae with the upper respiratory tract and tonsils .......................... 9



\footnotetext{
* Corresponding author: Koen.Chiers@UGent.be

This is an Open Access article distributed under the terms of the Creative Commons Attribution-Noncommercial License (http://creativecommons.org/licenses/by-nc/3.0/), which permits unrestricted use, distribution, and reproduction in any noncommercial medium, provided the original work is properly cited.
} 


\section{INTRODUCTION}

Porcine contagious pleuropneumonia is caused by the Gram-negative rod Actinobacillus pleuropneumoniae. This disease, which has been described world-wide, affects swine of all ages and has a serious impact on economy, ecology and animal welfare in the pig rearing industry [57]. Characterized by fibrinohemorrhagic necrotizing bronchopneumonia and fibrinous pleuritis, it often takes a fatal course. In vitro growth of $A$. pleuropneumoniae may be NAD dependent (biotype 1 strains) or NAD independent (biotype 2 strains) [87]. On the basis of the antigenic properties of the capsular polysaccharides and the cell wall lipopolysaccharides, A. pleuropneumoniae has been divided into 15 serotypes [17]. Although all serotypes can cause disease, differences in virulence exist [60]. In most herds, one serotype predominates, although several different serotypes have been demonstrated on one and the same farm [31]. Herds with a high traffic of animals have a higher risk of becoming infected with new serotypes.

Outbreaks of acute pleuropneumonia may occur in all age groups, but are mainly observed in fatteners. Animals of 12 weeks of age seem to be most susceptible [35]. In endemically infected herds, A. pleuropneumoniae may be detected in tonsillar samples taken from piglets less than 4 weeks of age, whereas its presence in lung tissue and the induction of lung lesions is often only seen from the age of 12-16 weeks onwards [31]. The pathogen is infrequently detected in nasal samples [31]. The factors allowing A. pleuropneumoniae to spread to and colonize lung tissue in pigs that carry the pathogen in their tonsils are not well-known. Risk factors such as stress, crowding and the moving and mixing of pigs, as well as adverse climatic conditions may be involved and contribute to the development and spread of the disease, thus affecting the rate of morbidity and mortality [85]. Concurrent or previous infections with other respiratory pathogens such as Mycoplasma hyopneumoniae [28, 77, 123] and Aujeszky's disease virus [100] can exacerbate the symptoms of pleuropneumonia.
However, this was not observed with an experimental PRRSV infection [91].

The pathogenesis of porcine contagious pleuropneumonia is complex, involving different virulence factors of the bacterium. This article aims to present an overview of the virulence factors of $A$. pleuropneumoniae that enable the pathogen to colonize the upper and lower respiratory tract, to persist there and to induce lesions.

\section{INTERACTIONS OF}

\section{A. PLEUROPNEUMONIAE WITH THE LOWER RESPIRATORY TRACT}

The lower respiratory tract is the site where A. pleuropneumoniae causes tissue damage leading to clinical disease and mortality. In general, the agent enters the lungs after inhalation as an aerosol. It colonises this tissue by binding to mucus, proteins and host cells, allowing multiplication and production of substances resulting in severe damage at these sites.

\subsection{Virulence factors involved in adhesion}

The bacteria bind preferentially to mucus, proteins and cells of the lower respiratory tract. The latter include ciliated cells of the terminal bronchioli and alveolar epithelial cells [44]. Several virulence factors may play a role in this adhesion phenomenon. In Table I, an overview is given of these factors and the corresponding genes.

Fimbriae are known to be involved in the adherence of several pathogens. Type 4 fimbriae have been demonstrated on A. pleuropneumoniae [44, 114, 116], fimbrial subunits have been purified [125] and the type 4 fimbrial structural gene (apfA) has been cloned and characterized [108]. Boekema et al. [18, 19] demonstrated that the production of type 4 fimbriae is induced by contact with epithelial cells in vitro and during lung infection in vivo, thus suggesting their possible role in adhesion.

Several studies have demonstrated the role of lipopolysaccharides in adhesion to tracheal mucus and porcine tracheal rings maintained in 
Table I. Virulence factors with confirmed or putative involvement in adhesion of A. pleuropneumoniae to the lower respiratory tract and the corresponding genes.

\begin{tabular}{|c|c|c|}
\hline (Putative) virulence factor & Gene & Reference \\
\hline $\begin{array}{l}\text { Type IV fimbriae (structural subunit, } \\
3 \text { biogenesis components) }\end{array}$ & apf $A B C D$ & $\begin{array}{c}{[18,19,44,108,114} \\
116,125]\end{array}$ \\
\hline Lipopolysaccharide biosynthesis & $\begin{array}{l}\text { galU, rmlC, } r f b N, \\
r f b P, r f b U, r f a E\end{array}$ & $\begin{array}{c}{[1,13,14,89,90} \\
92,93,97]\end{array}$ \\
\hline $\begin{array}{l}\text { Putative adhesin (YadA outer membrane } \\
\text { protein homologue) }\end{array}$ & $\mathrm{NA}^{*}$ & {$[33]$} \\
\hline $55 \mathrm{kDa}$ outer membrane protein & NA & [116] \\
\hline $60 \mathrm{kDa}$ outer membrane protein & NA & {$[46]$} \\
\hline $\begin{array}{l}\text { Putative adhesin (OmpA outer membrane } \\
\text { protein homologue) }\end{array}$ & рот $A$ & {$[8,53]$} \\
\hline $\begin{array}{l}\text { Fibronectin binding outer membrane } \\
\text { protein }\end{array}$ & comE1 & [80] \\
\hline Biofilm formation & 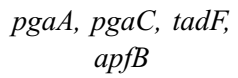 & {$[5,27,62,68-70,75]$} \\
\hline $\begin{array}{l}\text { Tight adherence protein (possible involved in } \\
\text { biofilm formation) }\end{array}$ & $\operatorname{tad} C, \operatorname{tad} D$ & {$[5,70]$} \\
\hline $\begin{array}{l}\text { Histone-like nucleoid structuring protein } \\
\text { (regulator of biofilm formation) }\end{array}$ & hns & [38] \\
\hline $\begin{array}{l}\text { Autotransporter serine protease } \\
\text { (involved in biofilm formation) }\end{array}$ & aas $P$ & {$[3,111]$} \\
\hline Putative autotransporter adhesin & $h s f$ & {$[5,8]$} \\
\hline $\begin{array}{l}\text { Putative fimbria-like protein } \\
\text { (possibly involved in microcolony formation) }\end{array}$ & flpD & {$[8]$} \\
\hline Putative fibronectin binding & tufA & [8] \\
\hline
\end{tabular}

* Information not available.

culture, as well as to frozen tracheal and lung sections [13, 14, 89, 90]. Glycosphingolipids were identified as receptors in respiratory epithelial cells [1]. Although the involvement of lipopolysaccharides in adherence has been questioned [97], and the adherence of $A$. pleuropneumoniae to lung epithelial cells has been found to be lipopolysaccharide-independent [18], a study using mutant strains with altered lipopolysaccharide structures confirmed their role in adhesion [93]. The oligosaccharide core of lipopolysaccharides seems to play a role in this phenomenon. Knocking out the $r f a E$ gene, which is involved in biosynthesis of lipopolysaccharides, resulted in a mutant strain that was no longer able to adhere [92].

Many outer membrane proteins have been identified in A. pleuropneumoniae [33]. Proteomic analysis demonstrated an outer membrane protein with similarity to YadA adhesin, which is involved in attachment and invasion of Yersinia [33]. An apparently unique outer membrane protein with a molecular weight of $55 \mathrm{kDa}$ was expressed in bacteria exhibiting a high degree of adhesion to alveolar epithelial cells [116]. The exact role of these proteins in adhesion, however, has not yet been elucidated. An outer membrane protein of $60 \mathrm{kDa}$ was found to be involved in adhesion to swine-lung collagen [46], and a small protein, comE1, was demonstrated to bind fibronectin [80]. A gene encoding a putative adhesin (pom $A$ ) was up-regulated in A. pleuropneumoniae recovered from necrotic lung tissue [8] and identified as important in virulence using signature-tagged mutagenesis [53].

Capsular polysaccharides are probably not involved in adherence, but rather mask, at least 
in part, the adhesive functions [98, 116]. In fact, capsular polysaccharide-associated genes are down-regulated during in vitro adhesion of A. pleuropneumoniae [5].

In vivo colonization of host tissues by bacteria is often mediated by biofilm formation. Biofilms are surface-associated colonies of bacteria embedded in an extracellular polymeric substance that enables autoaggregation and attachment to the underlying surface. Production of biofilms has been described in many A. pleuropneumoniae serotypes and is believed to play a role in colonization [68-70]. Izano et al. [62] identified poly- $N$-acetylglucosamine (PGA) as the major biofilm adhesin in A. pleuropneumoniae. Buettner et al. [27] demonstrated that a mutant strain deficient in biofilm formation was less virulent. The importance of a histone-like protein $\mathrm{H}-\mathrm{NS}$ in biofilm formation and virulence of $A$. pleuropneumoniae has been demonstrated by Dalai et al. [38]. Genes involved in biofilm formation were up-regulated in vitro in a malT (positive transcriptional regulator of the maltose regulon) mutant strain, mimicking a stringent gene expression response during nutrient deprivation [75]. This up-regulation was also observed after contact of $A$. pleuropneumoniae with porcine lung epithelial cells [5]. The role of biofilm formation in colonization needs further examination.

An $A$. pleuropneumoniae aas $P$ autotransporter serine protease-mutant strain showed decreased adhesion to abiotic surfaces, but still retained its full virulence, thus indicating that AasP is not necessary for full pathogenicity [111]. Since autotransporters can be involved in adhesin processing, the function of AasP could be to "fine-tune" the adhesion mechanisms. Until now, AasP has been demonstrated to be involved in cleavage and release of fragments of OmlA from the cell surface [3]. A putative autotransporter adhesin, similar to Haemophilus surface fibrils (hsf), was up-regulated in vitro after contact of $A$. pleuropneumoniae with porcine tracheal and lung cell lines [5] and in vivo in porcine necrotic lung tissue [8]. In Haemophilus influenzae serotype b, this is considered to be the major nonpilus adhesin and it was found to be associated with adherence to human epithelial cells. Whether this protein in
A. pleuropneumoniae plays a similar role, still needs to be elucidated.

Genes homologous to those playing a role in adhesion in other bacteria, such as $f l p D$ and tufA, have been demonstrated in $A$. pleuropneumoniae [8]. Their possible role in pathogenesis of porcine pleuropneumonia needs further investigation.

A. pleuropneumoniae is able to adhere to porcine surfactant proteins $\mathrm{B}$ and $\mathrm{C}$ in vitro, but the factors involved need to be elucidated (unpublished results). Nevertheless, this association might be the first step in colonization of alveoli, followed by adhesion to the plasma membrane of alveolar epithelial cells.

In conclusion, most probably several mechanisms and antigens are involved in the adhesion of $A$. pleuropneumoniae to the lower respiratory tract. A multiple-step binding process to epithelial cells has been proposed: A. pleuropneumoniae might first use low-affinity binding between the O-antigen of its cellwall lipopolysaccharides and phospholipids or short glycolipids on the host cell. Thereafter it might rely on the core oligosaccharide of its lipopolysaccharides and/or bacterial surface proteins $(55 \mathrm{kDa}$ outer membrane protein, type 4 fimbriae) to interact more avidly with other host cell receptors [65].

\subsection{Virulence factors involved in the acquisition of essential nutrients}

Confirmed or putative virulence factors involved in acquisition of nutrients and their corresponding genes are summarized in Table II.

In the lower respiratory tract, there is a limited supply of essential nutrients for the growth of bacteria. However, A. pleuropneumoniae has developed a number of features to overcome this impediment. For example, the pathogen can induce lysis of several cells, resulting in the release of nutrients into the environment. Lipopolysaccharides and secreted exotoxins (see below) may both be involved in this phenomenon.

Iron can be acquired by means of transferrin binding proteins $[6,8,12,37,54-56,88,96$, $112,121]$, siderophore receptors such as ferrichrome receptors $[41,78,79,103]$, and the binding of porcine hemoglobin by both 
Table II. Confirmed or putative virulence factors of $A$. pleuropneumoniae involved in acquisition of essential nutrients and their corresponding genes.

\begin{tabular}{|c|c|c|}
\hline (Putative) function & Gene & Reference \\
\hline Iron acquisition TonB 1 cluster & 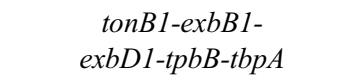 & $\begin{array}{c}{[5,6,8,37,40,54-56} \\
88,96,112,121]\end{array}$ \\
\hline Iron acquisition TonB 2 cluster & $\operatorname{exbB2-exbD2-tonB2}$ & {$[12,40]$} \\
\hline Ferric hydroxamate uptake & fhuA, fhuB, fhuC, fhuD & {$[41,70,78,79,103]$} \\
\hline $\begin{array}{l}\text { Hemoglobin-binding protein A } \\
\text { precursor }\end{array}$ & $h g b A$ & {$[4,5,15,40,103,107]$} \\
\hline $\begin{array}{l}\text { Putative nickel and cobalt periplasmic } \\
\text { permease system }\end{array}$ & cbiKLMQO & {$[5,22]$} \\
\hline Maltose regulon & $\begin{array}{c}\text { malEFG, malK-lamB-malM, } \\
\text { malT, malPQ }\end{array}$ & {$[39,75,76]$} \\
\hline Double stranded DNA binding & comE1 & {$[80]$} \\
\hline ferric transporter & $a f u B, a f u C$ & {$[70,75]$} \\
\hline Putative arginine/ornithine antiporter & $\operatorname{arcD}$ & {$[70]$} \\
\hline $\begin{array}{l}\text { Arginine transport system } \\
\text { permease protein }\end{array}$ & $\operatorname{artQ}$ & {$[40]$} \\
\hline $\begin{array}{l}\text { Branched-chain amino acid } \\
\text { transport system carrier protein }\end{array}$ & $\operatorname{brn} Q$ & {$[75,76]$} \\
\hline $\begin{array}{l}\text { Putative ferric enterobactin } \\
\text { transporter binding protein }\end{array}$ & fetB2 & [40] \\
\hline Iron-regulated outer membrane protein B & $\operatorname{frpB}$ & {$[75,76]$} \\
\hline Glycerol uptake facilitator and transporter & $\operatorname{glpF}, \operatorname{glp} T$ & {$[5,40,70,75,76]$} \\
\hline $\begin{array}{l}\text { D-ribose binding periplasmic and } \\
\text { transport protein }\end{array}$ & $r b s B, r b s D$ & {$[40,75,76]$} \\
\hline Peptide transport system permease protein & $\operatorname{sap} C$ & [40] \\
\hline Serine transporter & sdac & {$[75,76]$} \\
\hline Thiamine transport ATP-binding protein & thiQ & {$[70]$} \\
\hline Colicin transport proteins & tolQ, tolR & {$[5,76]$} \\
\hline Urea transport & $u t p$ & {$[22]$} \\
\hline $\begin{array}{l}\text { Iron (chelated) } \mathrm{ABC} \text { transporter, } \\
\text { periplasmic binding protein }\end{array}$ & $y f e A, y f e B, y f e C, y f e D$ & {$[5,40,75],[105]$} \\
\hline
\end{tabular}

lipopolysaccharides and outer membrane proteins $[4,15,103,107]$. Fatty acids of the lipid A of lipopolysaccharides were shown to bind porcine hemoglobin. Lipid A is normally anchored in the outer membrane. However, during formation of outer membrane blebs, this hydrophobic moiety is better exposed, allowing binding of hemoglobin [15]. During in vitro growth of $A$. pleuropneumoniae under iron restriction, several genes with confirmed or putative involvement in iron acquisition were up-regulated [40].

The mechanism for the uptake of nickel and cobalt is not known yet, although an operon for its transportation (cbiKLMQO) has been demonstrated [22].

An outer membrane protein of $42 \mathrm{kDa}$ may be involved in the uptake of maltose [39]. The genes involved in maltose regulation were demonstrated to be up-regulated in A. pleuropneumoniae exposed to bronchoalveolar lavage fluid [76]. Furthermore, a malT mutant strain exposed to bronchoalveolar fluid, expressed a gene profile resembling the stringent response during nutrient deprivation: up-regulation of genes involved in amino acid and nucleotide biosynthesis, biofilm formation, DNA transformation, and the stress response [75]. 
Table III. Virulence factors with confirmed or putative involvement in the induction of lesions by A. pleuropneumoniae and the corresponding genes.

\begin{tabular}{|c|c|c|c|}
\hline (Putative) virulence factor & Gene & Function & Reference \\
\hline $\begin{array}{l}\text { Pore forming RTX toxin I } \\
\text { (activator, structural unit, } \\
\text { secretion proteins) }\end{array}$ & apxICABD & $\begin{array}{l}\text { Strongly haemolytic; strongly } \\
\text { cytotoxic for alveolar macrophages } \\
\text { and neutrophils }\end{array}$ & $\begin{array}{l}{[42,50-52} \\
59,115]\end{array}$ \\
\hline $\begin{array}{l}\text { Pore forming RTX toxin II } \\
\text { (activator, structural unit) }\end{array}$ & apxIICA & $\begin{array}{l}\text { Weakly haemolytic; moderately } \\
\text { cytotoxic for alveolar macrophages } \\
\text { and neutrophils }\end{array}$ & $\begin{array}{c}{[42,43,50-52,} \\
59,102,115]\end{array}$ \\
\hline $\begin{array}{l}\text { Pore forming RTX toxin III } \\
\text { (activator, structural unit, } \\
\text { secretion proteins) }\end{array}$ & apxIIICABD & $\begin{array}{l}\text { Non-haemolytic, strongly } \\
\text { cytotoxic for alveolar } \\
\text { macrophages and neutrophils }\end{array}$ & $\begin{array}{c}{[42,43,50-52} \\
59,115]\end{array}$ \\
\hline $\begin{array}{l}\text { Putative pore forming } \\
\text { RTX toxin IV }\end{array}$ & apxIVA & Uncertain, required for full virulence & {$[8,32,74-76,101]$} \\
\hline $\begin{array}{l}\text { Lipopolysaccharide } \\
\text { biosynthesis }\end{array}$ & $\begin{array}{c}\text { galU, } r m l C, r f b N \\
r f b P, r f b U, r f a E\end{array}$ & $\begin{array}{l}\text { Enhanced effect of Apx toxins } \\
\text { on phagocytes, activation } \\
\text { of production of inflammatory cytokines, } \\
\text { induction of necrosis } \\
\text { in porcine lung epithelial cells }\end{array}$ & $\begin{array}{l}{[5,47,48} \\
93,94]\end{array}$ \\
\hline Proteases & NA* & $\begin{array}{l}\text { Degradation of porcine gelatin, } \\
\text { actin, hemoglobin }\end{array}$ & [81-83] \\
\hline
\end{tabular}

* Information not available.

It has been demonstrated that some bacteria can use nucleic acids as nutrients [49]. In A. pleuropneumoniae, a small protein, Com $\mathrm{E} 1$, is able to bind double stranded DNA and might be involved in this alternative pathway of nutrient metabolism [80].

In different experimental set-ups, similar genes as described above and several other genes encoding putative factors involved in nutrient uptake have been demonstrated (Tab. II) [5, 22, 40, 70, 75, 76, 105].

\subsection{Virulence factors involved in the induction of lesions}

The first, and probably most important, are the pore-forming exotoxins ApxI, ApxII and ApxIII [52] (Tab. III). All virulent A. pleuropneumoniae strains express 1 or 2 of these toxins. The genes involved in the production and secretion of Apx toxins have been well-characterized [52]. The secretion of Apx toxins results in lysis of alveolar epithelial cells, endo- thelial cells, red blood cells, neutrophils and macrophages [42, 43, 50, 51, 102, 115]. The adhesion of $A$. pleuropneumoniae to cells of the host may allow bacteria to release their toxins directly to the surface of the host cell membrane, resulting in the destruction of these cells, even in the presence of Apx toxin-neutralizing antibodies [59]. A fourth toxin (ApxIV), which has been demonstrated in all A. pleuropneumoniae strains [101], is essential for full virulence [74]. It is only expressed under in vivo conditions [32, 101]. The apxIVA gene was up-regulated during contact with bronchoalveolar fluid in vitro $[75,76]$ and expressed in necrotic porcine lung tissue in vivo [8]. Its exact role in pathogenesis, however, still needs to be elucidated.

Lipopolysaccharides also have the potential to cause damage to host cells. They are a major constituent of the outer membrane of Gram-negative bacteria. The lipopolysaccharides of $A$. pleuropneumoniae may contribute to the formation of lesions. They enhance the effects of Apx toxins on phagocytes, 
Table IV. Confirmed or putative virulence factors of A. pleuropneumoniae involved in avoiding the host's defense mechanism and the corresponding genes.

\begin{tabular}{|c|c|c|c|}
\hline (Putative) virulence factor & Gene & Function & Reference \\
\hline Proteases & NA* & $\begin{array}{l}\text { Degradation of porcine } \\
\text { IgA and IgG }\end{array}$ & {$[81,82]$} \\
\hline Apx toxins & see Table III & $\begin{array}{l}\text { Cytolysis of neutrophils and } \\
\text { macrophages, apoptosis } \\
\text { of alveolar macrophages, } \\
\text { impairment of macrophage } \\
\text { chemotactic and phagocytotic } \\
\text { function, intracellular survival }\end{array}$ & $\begin{array}{c}{[29,34,36,42} \\
43,110]\end{array}$ \\
\hline Urease & $\begin{array}{l}\text { ureABC, } \\
\text { ureDEFG }\end{array}$ & $\begin{array}{l}\text { Intracellular survival, } \\
\text { impairment of } \\
\text { macrophage function, } \\
\text { toxic for macrophages }\end{array}$ & {$[20,21]$} \\
\hline $\begin{array}{l}\text { Carbohydrates in capsule } \\
\text { and lipopolysaccharides }\end{array}$ & NA & Intracellular survival & [16] \\
\hline Heat-shock protein & dnaK & Intracellular survival & [53] \\
\hline $\mathrm{Cu}-\mathrm{Zn}$ superoxide dismutase & $\operatorname{sod} C$ & Intracellular survival & {$[71,104]$} \\
\hline Capsular polysaccharide & cpxDCBA & Antiphagocytic, serum resistance & $\begin{array}{c}{[8,11,61,66,75,} \\
97-99,113,118-120]\end{array}$ \\
\hline Regulator of the maltose regulon & malT & Serum resistance & {$[75]$} \\
\hline
\end{tabular}

* Information not available.

activate the production of inflammatory cytokines $[47,48,93]$ and induce necrosis in porcine lung epithelial cell lines via binding to Toll-like receptors [5]. Direct binding of lipopolysaccharide outer core (GalNAc-Gal II-Gal I) to ApxI and II toxins might explain the enhanced cytotoxicity [94].

Other factors that may play a role in tissue damage are the different proteases secreted by A. pleuropneumoniae, which degrade porcine gelatine, actin and hemoglobin [81-83].

\subsection{Virulence factors involved in avoiding the host's defense mechanisms}

The effectiveness of the host's defense against pulmonary bacterial infections depends on the rapid clearance of the microorganisms from the respiratory tract [23]. Besides nonspecific factors such as the ciliary beat, the cough reflex and mucus clearance, the innate pulmonary immunity is composed of a cellular portion (airway and alveolar epithelial cells, resident and recruited leukocytes) and a humoral component (antimicrobial products secreted in the epithelial lining fluid) [124]. A. pleuropneumoniae, however, possesses several properties that enable it to avoid the host's immune system (Tab. IV).

A. pleuropneumoniae can survive a $30 \mathrm{~min}$ exposure to bronchoalveolar lavage fluid while $70 \%$ of Escherichia coli cells were killed [76]. Genes involved in cell envelope, DNA, and protein biosynthesis as well as those playing a role in the bacterium's energy metabolism were most frequently up-regulated. Genes encoding proteins for co-factor biosynthesis, toxin production and secretion and trafficking of ions and biomolecules were also up-regulated while genes encoding proteins involved in protein folding and stabilization, nucleotide biosynthesis, and mobile elements were down-regulated $[75,76]$.

The pathogen secretes proteases that degrade porcine $\operatorname{IgA}$ and $\operatorname{IgG}[81,82]$. It is not known whether these proteases impair the host's defense against bacterial adhesion, toxins or in vivo opsonization. 
ApxI, II and III toxins have lethal effects on neutrophils and macrophages [36, 42, 43] and may thus also play a role in impairing the host's defenses. Chien et al. [29] demonstrated that ApxI induces apoptosis of pulmonary alveolar macrophages. At sublytic dose, the toxins impair macrophage chemotactic and phagocytic functions [110].

An A. pleuropneumoniae serotype 2 mutant strain, deficient in production of ApxII and ApxIII, was still capable of damaging porcine alveolar macrophages in vitro [36]. Therefore, other cytotoxic factors could be involved, such as ammonia. Indeed, urease activity is present in all A. pleuropneumoniae serotypes and ammonia may act synergistically with Apx toxins. This may contribute to cytotoxicity, as well as to impaired macrophage function [20, 21].

After phagocytosis, A. pleuropneumoniae can survive for up to $90 \mathrm{~min}$ in macrophages [34]. Several factors may contribute to this intracellular survival, such as high molecular-weight surface carbohydrates present in capsule and lipopolysaccharides [16], secretion of Apx toxins [34], heat-shock proteins [53], ammonia [21] and copper-zinc superoxide dismutase [71, 104].

Capsular polysaccharides and/or lipopolysaccharides of $A$. pleuropneumoniae exert antiphagocytic properties $[61,97-99,113,118$, 120]. Furthermore, Ward et al. [118] demonstrated that capsulated (A. pleuropneumoniae serotype 5a) strains were resistant to complement-mediated killing, whereas non-capsulated strains were killed. However, this may be serotype or strain dependent since a serotype 1 capsule mutant still was resistant [98]. A malT mutant strain was not able to survive following incubation with porcine serum. This may be due to changes in its cell surface polysaccharide composition [75]. Anyway, virulence of an A. pleuropneumoniae strain is influenced by the amount and type of capsular polysaccharides as well as by its mechanism of expression [11]. The genetic organization of the capsule biosynthesis region of several A. pleuropneumoniae serotypes has been described [66, 119 , 120]. Several of these genes were up-regulated in necrotic lung tissue [8] and during contact with bronchoalvealar lavage fluid [75].
Finally, biofilm formation may also increase resistance to the host's immune system by interfering with the macrophage phagocytic activity so as to keep the antibodies from reaching the surface of the bacterial cells and thus decreasing these cells' sensitivity to killing by polymorphonuclear leukocytes [25, 45]. However, this still needs to be demonstrated in A. pleuropneumoniae.

\subsection{Virulence factors involved in persistence}

A summary of virulence factors possibly involved in persistence and the corresponding genes is presented in Table V.

A. pleuropneumoniae may persist during prolonged periods of time in necrotic lung tissue [8]. At this site, oxygen availability is low. In such conditions, A. pleuropneumoniae expresses a global regulatory gene $(h l y X)$ that activates production of several enzymes such as dimethyl sulfoxide reductase and aspartate ammonia lyase, which enable anaerobic respiration of the bacterium [7, 9, 10, 64]. Expression of genes encoding hemoglobin-binding proteins and dimethylsulfoxide reductase is also stimulated $[7,8]$.

Sheehan et al. [105] demonstrated that several genes are required for the survival of A. pleuropneumoniae in its porcine host, including the genes involved in biosynthesis of cell surface structures, energy metabolism, nutrient uptake and stress response. In addition, Baltes et al. [10] demonstrated the up-regulation of different genes involved in nutrient transport, stress response, energy metabolism and the synthesis of nucleic acids or cellular components in the chronic stage of the disease.

Flagella and motility in A. pleuropneumoniae have been demonstrated, but their role in pathogenesis and survival in the host has not yet been elucidated [84].

In lung fluid, essential branched-chain amino acids, such as isoleucine, leucine, and valine are limited. Subashchandrabose et al. [109] demonstrated that a gene encoding an enzyme (acetohydroxyacid synthase) required for branched-chain amino acid biosynthesis was expressed in A. pleuropneumoniae in vivo, 
Table V. Virulence factors of $A$. pleuropneumoniae with putative involvement in persistence in the upper respiratory tract and the corresponding genes.

\begin{tabular}{lcc}
\hline (Putative) virulence factor & Gene & Reference \\
\hline Aspartate ammonia-lyase & $a s p A$ & {$[64]$} \\
Global anaerobic regulator & $h l y X$ & {$[7,9,10]$} \\
Dimethylsulfoxide reductase & $d m s A, \operatorname{dms} B, d m s C$ & {$[7,8]$} \\
Hemoglobin binding protein & $h g b A$ & {$[8]$} \\
Capsular polysaccharide export & $c p x B, c p x C, c p x D$ & {$[10,105]$} \\
Lipopolysaccharide biosynthesis & $g a l U, r m l C, r f b N, r f b P, r f b U$ & {$[105]$} \\
Autotransporter serine protease & $a a s P$ & {$[10]$} \\
Stress protein & $d n a K$ & {$[10]$} \\
Branched-chain amino acid biosynthesis & $I l v I$ & {$[109]$} \\
Aerobic respiration control protein & $a c r A$ & {$[26]$} \\
\hline
\end{tabular}

and that this was necessary for survival and full virulence.

In a recent study, Buettner et al. [26] demonstrated the importance of ArcA (a global cytosolic response regulator facilitating metabolic adaptation to anaerobicity and changing redox potential) in biofilm formation and autoaggregation, and hypothetized that ArcA plays an essential role in respiratory tract persistence of A. pleuropneumoniae. Biofilm formation does not only allow colonization. Bacteria in a biofilm also often exhibit increased resistance to antimicrobial agents, making it difficult to eradicate [68]. However, no genetic data are yet available confirming this hypothesis.

ComE1 has been shown to play a major role in natural transformation of some A. pleuropneumoniae strains, which could allow adaptation of the bacterium and persistence in the host $[24,80]$.

Taken together, all this evidence indicates that the pathogen has a capacity to adapt to different environmental conditions enabling longtime survival in the host.

\section{INTERACTIONS OF}

\section{A. PLEUROPNEUMONIAE WITH THE UPPER RESPIRATORY TRACT AND TONSILS}

A. pleuropneumoniae may colonize the tonsils and, albeit to a lesser extent, the nasal mucosa in the presence or absence of lung colonization $[63,86,106]$. Indeed, in some subclinical infections, the bacterium does not enter the lungs but persists in the tonsils, thus resulting in symptom-free carriers $[31,58,67,106$, $117,122]$. In such animals, antibodies against A. pleuropneumoniae are usually not detected $[31,73,106]$.

Although healthy animals may carry A. pleuropneumoniae in their nose, information on the mechanisms by which the bacterium resides in this organ is lacking.

Adhesion of $A$. pleuropneumoniae to tracheal crude mucus has been well documented and lipopolysaccharides were found to be involved [14]. Since the epithelium of tonsils is also covered by a mucus layer, such interaction may constitute a first step in colonization. However, mucus (and attached bacteria) is continuously being cleared, and therefore bacteria need other mechanisms to persist. Such a mechanism could involve a specific binding of bacteria to epithelial cells. Indeed, A. pleuropneumoniae adheres to surface and crypt tonsillar epithelial cells. This was presumed to be a specific interaction since the bacteria were closely associated with microprojections of epithelial cells [30]. A similar two-step mechanism has been described in the pathogenesis of Streptococcus pyogenes pharyngotonsillitis in humans [72]. Adherent biofilms have been shown to be associated with chronic tonsillitis in children [2]. Whether 
biofilm formation contributes to the colonization of A. pleuropneumoniae in porcine tonsils, however, still needs to be elucidated.

There is speculation that tonsillar crypts are the niche in which A. pleuropneumoniae may persist [30]. The mechanism by which this happens is not known, but since bacteria are localized in the deeper parts of the crypts and tonsils are covered by mucus, oxygen availability is probably low at these sites. Therefore, similar mechanisms as have been proposed for persistence in necrotic lung tissue may play a role $[7,64]$. For instance, it was demonstrated that a $h l y X$ (gene involved in anaerobic respiration) mutant is unable to persist in tonsils [9]. In addition, maltodextrin carbohydrate metabolism also might play a role in tonsillar persistence, since all serotypes of $A$. pleuropneumoniae possess the maltose-regulon genes and maltodextrins can be found in the oropharynx [75].

In natural $A$. pleuropneumoniae infections, the tonsils and nasal mucosa are not routinely examined for the presences of lesions, and information on this subject is therefore lacking. However, experimental inoculation of tonsils in gnotobiotic piglets resulted in vacuolization, localized disruption and necrosis of the superficial epithelium with subsequent hyperemia and neutrophilic inflammation [30]. The factors involved in these findings were not examined. Since the events are very similar to those inducing lung lesions, it is tentative to speculate that similar virulence factors may play a role.

\section{CONCLUSIONS}

The virulence factors and their corresponding genes involved in lung colonization and induction of lung lesions by A. pleuropneumoniae have been thoroughly studied and some have been well characterized. This information has been used to design new vaccines that induce partial protection against the disease $[60,95]$. A. pleuropneumoniae has the capacity to persist in its host during prolonged periods of time, and this factor plays a role in the spread of the infection. In addition to persistence in lung lesions, the colonization of the upper respiratory tract and of the tonsils, in particular, is important for long-term persistent asymptomatic infection. Information on virulence factors involved in tonsillar and nasal cavity colonization and persistence is scarce.

The availability of full genomes of A. pleuropneumoniae should allow new approaches to further clarify the role of selective genes in the pathogenesis of porcine pleuropneumonia and persistence of the micro-organism in its host. It may also help to elucidate cellular mechanisms and pathways involved in these processes.

Acknowledgements. The authors thank Marleen Foubert for her excellent secretarial assistance.

\section{REFERENCES}

[1] Abul-Milh M., Paradis S.E., Dubreuil J.D., Jacques M., Binding of Actinobacillus pleuropneumoniae lipopolysaccharides to glycosphingolipids evaluated by thin-layer chromatography, Infect. Immun. (1999) 67:4983-4987.

[2] Al-Mazrou K.A., Al-Khattaf A.S., Adherent biofilms in adenotonsillar diseases in children, Arch. Otolaryngol. Head Neck Surg. (2008) 134:20-23.

[3] Ali T., Oldfield N.J., Wooldridge K.G., Turner D.P., Ala'Aldeen D.A.A., Functional characterization of AasP, a maturation protease autotransporter protein of Actinobacillus pleuropneumoniae, Infect. Immun. (2008) 76:5608-5614.

[4] Archambault M., Rioux S., Jacques M., Evaluation of the hemoglobin-binding activity of Actinobacillus pleuropneumoniae using fluorescein-labeled pig hemoglobin and flow cytometry, FEMS Microbiol. Lett. (1999) 173:17-25.

[5] Auger E., Deslandes V., Ramjeet M., Contreras I., Nash J.H.E., Harel J., et al., Host-pathogen interactions of Actinobacillus pleuropneumoniae with porcine lung and tracheal epithelial cells, Infect. Immun. (2009) 77:1426-1441.

[6] Baltes N., Hennig-Pauka I., Gerlach G.F., Both transferrin binding proteins are virulence factors in Actinobacillus pleuropneumoniae serotype 7 infection, FEMS Microbiol. Lett. (2002) 209:283-287.

[7] Baltes N., Hennig-Pauka I., Jacobsen I., Gruber A.D., Gerlach G.F., Identification of dimethyl sulfoxide reductase in Actinobacillus pleuropneumoniae and its role in infection, Infect. Immun. (2003) 71:6784-6792.

[8] Baltes N., Gerlach G.F., Identification of genes transcribed by Actinobacillus pleuropneumoniae 
in necrotic porcine lung tissue by using selective capture of transcribed sequences, Infect. Immun. (2004) 72:6711-6716.

[9] Baltes N., N'diaye M., Jacobsen I.D., Maas A., Buettner F.F.R., Gerlach G., Deletion of the anaerobic regulator HlyX causes reduced colonization and persistence of Actinobacillus pleuropneumoniae in the porcine respiratory tract, Infect. Immun. (2005) 73:4614-4619.

[10] Baltes N., Buettner F.F.R., Gerlach G.F., Selective capture of transcribed sequences (SCOTS) of Actinobacillus pleuropneumoniae in the chronic stage of disease reveals an HlyX-regulated autotransporter protein, Vet. Microbiol. (2007) 123:110-121.

[11] Bandara A.B., Lawrence M.L., Veit H.P., Inzana T.J., Association of Actinobacillus pleuropneumoniae capsular polysaccharide with virulence in pigs, Infect. Immun. (2003) 71:3320-3328.

[12] Beddek A.J., Sheehan B.J., Bossé J.T., Rycroft A.N., Kroll J.S., Langford P.R., Two TonB systems in Actinobacillus pleuropneumoniae: their roles in iron acquisition and virulence, Infect. Immun. (2004) 2:701-708.

[13] Bélanger M., Dubreuil D., Harel J., Girard C., Jacques M., Role of lipopolysaccharides in adherence of Actinobacillus pleuropneumoniae to porcine tracheal rings, Infect. Immun. (1990) 58:3523-3530.

[14] Bélanger M., Dubreuil D., Jacques M., Proteins found within porcine respiratory tract secretions bind lipopolysaccharides of Actinobacillus pleuropneumoniae, Infect. Immun. (1994) 62:868-873.

[15] Bélanger M., Bégin C., Jacques M., Lipopolysaccharides of Actinobacillus pleuropneumoniae bind pig hemoglobin, Infect. Immun. (1995) 63:656-662.

[16] Bilinski T., Oxygen toxicity and microbial evolution, Biosystems (1991) 24:305-312.

[17] Blackall P.J., Klaasen H.L.B.M., Bosch H., Kuhnert P., Frey J., Proposal of a new serovar of Actinobacillus pleuropneumoniae: serovar 15, Vet. Microbiol. (2002) 84:47-52.

[18] Boekema B.K.H.L., Stockhofe-Zurwieden N., Smith H.E., Kamp E.M., van Putten J.P., Verheijden J.H., Adherence of Actinobacillus pleuropneumoniae to primary cultures of porcine lung epithelial cells, Vet. Microbiol. (2003) 93:133-144.

[19] Boekema B.K.H.L., Van Putten J.P.M., Stockhofe-Zurwieden N., Smith H.E., Host cell contactinduced transcription of the type IV fimbria gene cluster of Actinobacillus pleuropneumoniae, Infect. Immun. (2004) 72:691-700.
[20] Bossé J.T., MacInnes J.I., Genetic and biochemical analyses of Actinobacillus pleuropneumoniae urease, Infect. Immun. (1997) 65:4389-4394.

[21] Bossé J.T., MacInnes J.I., Urease activity may contribute to the ability of Actinobacillus pleuropneumoniae to establish infection, Can. J. Vet. Res. (2000) 64:145-150.

[22] Bossé J.T., Gilmour H.D., MacInnes J.I., Novel genes affecting urease activity in Actinobacillus pleuropneumoniae, J. Bacteriol. (2001) 183:1242-1247.

[23] Bossé J.T., Janson H., Sheehan B.J., Beddek A.J., Rycroft A.N., Kroll J.S., Langford P.R., Actinobacillus pleuropneumoniae: pathobiology and pathogenesis of infection, Microbes Infect. (2002) 4:225-235.

[24] Bossé J.T., Sinha S., Schippers T., Kroll J.S., Redfield R.J., Langford P.R., Natural competence in strains of Actinobacillus pleuropneumoniae, FEMS Microbiol. Lett. (2009) 298:124-130.

[25] Boyen F., Eeckhaut V., Van Immerseel F., Pasmans F., Ducatelle R., Haesebrouck F., Quorum sensing in veterinary pathogens: mechanisms, clinical importance and future perspectives, Vet. Microbiol. (2009) 135:187-195.

[26] Buettner F.F.R., Bendallah I.M., Bossé J.T., Dreckmann K., Nash J.H.E., Langford P.R., Gerlach G.F., Analysis of the Actinobacillus pleuropneumoniae $\operatorname{ArcA}$ regulon identifies fumarate reductase as a determinant of virulence, Infect. Immun. (2008) 76:2284-2295.

[27] Buettner F.F.R., Maas A., Gerlach G.F., An Actinobacillus pleuropneumoniae arcA deletion mutant is attenuated and deficient in biofilm formation, Vet. Microbiol. (2008) 127:106-115.

[28] Caruso J., Ross R., Effects of Mycoplasma hyopneumoniae and Actinobacillus (Haemophilus) pleuropneumoniae infections on alveolar macrophage functions in swine, Am. J. Vet. Res. (1990) 51: 227-231.

[29] Chien M.S., Chan Y.Y., Chen Z.W., Wu C.M., Liao J.W., Chen T.H., et al., Actinobacillus pleuropneumoniae serotype 10 derived ApxI induces apoptosis in porcine alveolar macrophages, Vet. Microbiol. (2009) 135:327-333.

[30] Chiers K., Haesebrouck F., van Overbeke I., Charlier G., Ducatelle R., Early in vivo interactions of Actinobacillus pleuropneumoniae with tonsils of pigs, Vet. Microbiol. (1999) 68:301-306.

[31] Chiers K., Donné E., Overbeke I.V., Ducatelle R., Haesebrouck F., Actinobacillus pleuropneumoniae infections in closed swine herds: infection patterns and 
serological profiles, Vet. Microbiol. (2002) 85: 343-352.

[32] Cho W.S., Chae C., Expression of the apxIV gene in pigs naturally infected with Actinobacillus pleuropneumoniae, J. Comp. Pathol. (2001) 125:3440.

[33] Chung J.W., Ng-Thow-Hing C., Budman L.I., Gibbs B.F., Nash J.H.E., Jacques M., Coulton J.W., Outer membrane proteome of Actinobacillus pleuropneumoniae: LC-MS/MS analyses validate in silico predictions, Proteomics (2007) 7:1854-1865.

[34] Cruijsen T.L.M., van Leengoed L.A.M.G., Dekker-Nooren T.C.E.M., Schoevers E.J., Verheijden J.H.M., Phagocytosis and killing of Actinobacillus pleuropneumoniae by alveolar macrophages and polymorphonuclear leukocytes isolated from pigs, Infect. Immun. (1992) 60:4867-4871.

[35] Cruijsen T.L.M., van Leengoed L.A.M.G., Kamp E.M., Bartelse A., Korevaar A., Verheijden J.H.M., Susceptibility to Actinobacillus pleuropneumoniae infection in pigs from an endemically infected herd is related to the presence of toxin-neutralizing antibodies, Vet. Microbiol. (1995) 47:219-228.

[36] Cullen J.M., Rycroft A.N., Phagocytosis by pig alveolar macrophages of Actinobacillus pleuropneumoniae serotype-2 mutant strains defective in hemolysin-II (ApxII) and pleurotoxin (ApxIII), Microbiology (1994) 140:237-244.

[37] Daban M., Medrano A., Querol E., Cloning, sequencing and expression of the transferrin-binding protein 1 gene from Actinobacillus pleuropneumoniae, Biochem. J. (1996) 315:257-264.

[38] Dalai B., Zhou R., Wan Y., Kang M.S., Li L., Li T.T., et al., Histone-like protein H-NS regulates biofilm formation and virulence of Actinobacillus pleuropneumoniae, Microb. Pathog. (2009) 46:128134.

[39] Deneer H.G., Potter A.A., Identification of a maltose-inducible major outer membrane protein in Actinobacillus pleuropneumoniae, Microb. Pathog. (1989) 6:425-432.

[40] Deslandes V., Nash J.H.E., Harel J., Coulton J.W., Jacques M., Transcriptional profiling of Actinobacillus pleuropneumoniae under iron-restricted conditions, BMC Genomics (2007) 8:72.

[41] Diarra M.S., Dolence J., Dolence E.K., Darwish I., Miller M.J., Malouin F., Jacques M., Growth of Actinobacillus pleuropneumoniae is promoted by exogenous hydroxamate and catechol siderophores, Appl. Environ. Microbiol. (1996) 62:853-859.

[42] Dom P., Haesebrouck F., De Baetselier P., Stimulation and suppression of the oxygenation activity of porcine pulmonary alveolar macrophages by Actinobacillus pleuropneumoniae and its metabolites, Am. J. Vet. Res. (1992) 53:1113-1118.

[43] Dom P., Haesebrouck F., Kamp E., Smits M., Influence of Actinobacillus pleuropneumoniae serotype 2 and its cytolysins on porcine neutrophil chemiluminescence, Infect. Immun. (1992) 60:43284334.

[44] Dom P., Haesebrouck F., Ducatelle R., Charlier G., In vivo association of Actinobacillus pleuropneumoniae serotype 2 with the respiratory epithelium of pigs, Infect. Immun. (1994) 62:1262-1267.

[45] Donlan R.M., Costerton J.W., Biofilms: survival mechanisms of clinically relevant microorganisms, Clin. Microbiol. Rev. (2002) 15:167-193.

[46] Enriquez-Verdugo I., Guerrero A.L., Serrano J.J., Godinez D., Rosales J.L., Tenorio V., de la Garza M., Adherence of Actinobacillus pleuropneumoniae to swine-lung collagen, Microbiology (2004) 150:23912400

[47] Fenwick B., Osburn B., Immune responses to the lipopolysaccharides and capsular polysaccharides of Haemophilus pleuropneumoniae in convalescent and immunized pigs, Infect. Immun. (1986) 54:575-582.

[48] Fenwick B., Henry S., Porcine pleuropneumonia, J. Am. Vet. Med. Assoc. (1994) 204:1334-1340.

[49] Finkel S.E., Kolter R., DNA as a nutrient novel role for bacterial competence gene homologs, J. Bacteriol. (2001) 183:6288-6293.

[50] Frey J., Bosse J.T., Chang Y.F., Cullen J.M., Fenwick B., Gerlach G.F., et al., Actinobacillus pleuropneumoniae RTX-toxins: uniform designation of haemolysins, cytolysins, pleurotoxin and their genes, J. Gen. Microbiol. (1993) 139:1723-1728.

[51] Frey J., Kuhn R., Nicolet J., Association of the CAMP phenomenon in Actinobacillus pleuropneumoniae with the RTX toxins ApxI, ApxII and ApxIII, FEMS Microbiol. Lett. (1994) 124:245-251.

[52] Frey J., Virulence in Actinobacillus pleuropneumoniae and RTX toxins, Trends Microbiol. (1995) 3:257-261.

[53] Fuller T.E., Martin S., Teel J.F., Alaniz G.R., Kennedy M.J., Lowery D.E., Identification of Actinobacillus pleuropneumoniae virulence genes using signature-tagged mutagenesis in a swine infection model, Microb. Pathog. (2000) 29:39-51.

[54] Gerlach G.F., Klashinsky S., Anderson C., Potter A.A., Willson P.J., Characterization of two genes encoding distinct transferrin-binding proteins in different Actinobacillus pleuropneumoniae isolates, Infect. Immun. (1992) 60:3253-3261. 
[55] Gonzalez G.C., Caamano D.L., Schryvers A.B., Identification and characterization of a porcine-specific transferrin receptor in Actinobacillus pleuropneumoniae, Mol. Microbiol. (1990) 4:1173-1179.

[56] Gonzalez G.C., Yu R.H., Rosteck P.R. Jr, Schryvers A.B., Sequence, genetic analysis, and expression of Actinobacillus pleuropneumoniae transferrin receptor genes, Microbiology (1995) 141:24052416.

[57] Gottschalk M., Taylor D.J., Actinobacillus pleuropneumoniae, in: Straw B.E., Zimmerman J.J., D'Allaire S., Taylor D.J. (Eds.), Diseases of swine, Blackwell Publishing Professional, Ames, Iow, USA, 2006, pp. 563-576.

[58] Gram T., Ahrens P., Nielsen J.P., Evaluation of a PCR for detection of Actinobacillus pleuropneumoniae in mixed bacterial cultures from tonsils, Vet. Microbiol. (1996) 51:95-104.

[59] Haesebrouck F., Chiers K., Van Overbeke I., Ducatelle R., Actinobacillus pleuropneumoniae infections in pigs: the role of virulence factors in pathogenesis and protection, Vet. Microbiol. (1997) 58:239-249.

[60] Haesebrouck F., Pasmans F., Chiers K., Maes D., Ducatelle R., Decostere A., Efficacy of vaccines against bacterial diseases in swine: what can we expect?, Vet. Microbiol. (2004) 100:255-268.

[61] Inzana T.J., Ma J., Workman T., Gogolewski R.P., Anderson P., Virulence properties and protective efficacy of the capsular polymer of Haemophilus (Actinobacillus) pleuropneumoniae serotype 5, Infect. Immun. (1988) 56:1880-1889.

[62] Izano E.A., Sadovskaya I., Vinogradov E., Mulks M.H., Velliyagounder K., Ragunath C., et al., Poly-N-acetylglucosamine mediates biofilm formation and antibiotic resistance in Actinobacillus pleuropneumoniae, Microb. Pathog. (2007) 43:1-9.

[63] Jacobsen M.J., Nielsen J.P., Development and evaluation of a selective and indicative medium for isolation of Actinobacillus pleuropneumoniae from tonsils, Vet. Microbiol. (1995) 47:191-197.

[64] Jacobsen I., Hennig-Pauka I., Baltes N., Trost M., Gerlach G.F., Enzymes involved in anaerobic respiration appear to play a role in Actinobacillus pleuropneumoniae virulence, Infect. Immun. (2005) 73:226-234.

[65] Jeannotte M.E., Abul-Milh M., Dubreuil J.D., Jacques M., Binding of Actinobacillus pleuropneumoniae to phosphatidylethanolamine, Infect. Immun. (2003) 71:4657-4663.

[66] Jessing S.G., Ahrens P., Inzana T.J., Angen O., The genetic organisation of the capsule biosynthesis region of Actinobacillus pleuropneumoniae serotypes 1, 6, 7, and 12, Vet. Microbiol. (2008) 129:350-359.

[67] Jolie R.A.V., Mulks M.H., Thacker B.J., Antigenic differences within Actinobacillus pleuropneumoniae serotype 1, Vet. Microbiol. (1994) 38:329349.

[68] Kaplan J.B., Velliyagounder K., Ragunath C., Rohde H., Mack D., Knobloch J.K.M., Ramasubbu $\mathrm{N}$., Genes involved in the synthesis and degradation of matrix polysaccharide in Actinobacillus actinomycetemcomitans and Actinobacillus pleuropneumoniae biofilms, J. Bacteriol. (2004) 186:8213-8220.

[69] Kaplan J.B., Mulks M.H., Biofilm formation is prevalent among field isolates of Actinobacillus pleuropneumoniae, Vet. Microbiol. (2005) 108:89-94.

[70] Labrie J., Pelletier-Jacques G., Deslandes V., Ramjeet M., Auger E., Nash J.H.E., Jacques M., Effects of growth conditions on biofilm formation by Actinobacillus pleuropneumoniae, Vet. Res. (2010) 41:03.

[71] Langford P.R., Loynds B.M., Kroll J.S., Cloning and molecular characterization of $\mathrm{Cu}, \mathrm{Zn}$ superoxide dismutase from Actinobacillus pleuropneumoniae, Infect. Immun. (1996) 64:5035-5041.

[72] Lilja M., Silvola J., Räisänen S., Stenfors L.E., Where are the receptors for Streptococcus pyogenes located on the tonsillar surface epithelium?, Int. J. Pediatr. Otorhinolaryngol. (1999) 50:37-43.

[73] Little T.W.A., Haemophilus infection in pigs, Vet. Rec. (1970) 7:399-402.

[74] Liu J.L., Chen X., Tan C., Guo Y., Chen Y., Fu S.L., et al., In vivo induced RTX toxin ApxIVA is essential for the full virulence of Actinobacillus pleuropneumoniae, Vet. Microbiol. (2009) 137:282289.

[75] Lone A.G., Deslandes V., Nash J.H.E., Jacques M., MacInnes J.I., malT knockout mutation invokes a stringent type gene-expression profile in Actinobacillus pleuropneumoniae in bronchoalveolar fluid, BMC Microbiol. (2009) 9:195.

[76] Lone A.G., Deslandes V., Nash J.H.E., Jacques M., MacInnes J.I., Modulation of gene expression in Actinobacillus pleuropneumoniae exposed to bronchoalveolar fluid, PLoS ONE (2009) 4:e6139.

[77] Marois C., Gottschalk M., Morvan H., Fablet C., Madec F., Kobisch M., Experimental infection of SPF pigs with Actinobacillus pleuropneumoniae serotype 9 alone or in association with Mycoplasma hyopneumoniae, Vet. Microbiol. (2009) 135:283-291.

[78] Mikael L.G., Pawelek P.D., Labrie J., Sirois M., Coulton J.W., Jacques M., Molecular cloning and 
characterization of the ferric hydroxamate uptake $(f h u)$ operon in Actinobacillus pleuropneumoniae, Microbiology (2002) 148:2869-2882.

[79] Mikael L.G., Srikumar R., Coulton J.W., Jacques M., fhuA of Actinobacillus pleuropneumoniae encodes a ferrichrome receptor but is not regulated by iron, Infect. Immun. (2003) 71:2911-2915.

[80] Mullen L.M., Bossé J.T., Nair S.P., Ward J.M., Rycroft A.N., Robertson G., et al., Pasteurellaceae ComE1 proteins combine the properties of fibronectin adhesins and DNA binding competence proteins, PLoS ONE (2008) 3:e3991.

[81] Negrete-Abascal E., Tenorio V.R., Serrano J.J., Garcia C., de la Garza M., Secreted proteases from Actinobacillus pleuropneumoniae serotype 1 degrade porcine gelatin, hemoglobin and immunoglobulin A, Can. J. Vet. Res. (1994) 58:83-86.

[82] Negrete-Abascal E., Tenorio V.R., Guerrero A.L., Garcia R.M., Reyes M.E., de la Garza M., Purification and characterization of a protease from Actinobacillus pleuropneumoniae serotype 1, an antigen common to all the serotypes, Can. J. Vet. Res. (1998) 62:183-190.

[83] Negrete-Abascal E., Garcia R.M., Reyes M.E., Godinez D., de la Garza M., Membrane vesicles released by Actinobacillus pleuropneumoniae contain proteases and Apx toxins, FEMS Microbiol. Lett. (2000) 191:109-113.

[84] Negrete-Abascal E., Reyes M.E., Garcia R.M., Vaca S., Giron J.A., Garcia O., et al., Flagella and motility in Actinobacillus pleuropneumoniae, J. Bacteriol. (2003) 185:664-668.

[85] Nicolet J., Bacteriology and epidemiology of Haemophilus pleuropneumoniae, in: Proceedings of the American Association of Swine Practitioners, 1985, pp. 7-11.

[86] Nicolet J., Actinobacillus pleuropneumoniae, in: Leman A.D., Straw B., Mengeling W.L., D’Allaire S., Taylor D.J. (Eds.), Diseases of Swine, Iowa State University Press, Ames, 1992, pp. 401-408.

[87] Niven D.F., Levesque M., V-Factor-dependent growth of Actinobacillus pleuropneumoniae biotype2, Int. J. Syst. Bacteriol. (1988) 38:319-320.

[88] Niven D.F., Donga J., Archibald F.S., Responses of Haemophilus pleuropneumoniae to iron restriction: changes in the outer membrane protein profile and the removal of iron from porcine transferrin, Mol. Microbiol. (1989) 3:1083-1089.

[89] Paradis S.E., Dubreuil D., Rioux S., Gottschalk M., Jacques M., High-molecular-mass lipopolysaccharides are involved in Actinobacillus pleuropneumoniae adherence to porcine respiratory tract cells, Infect. Immun. (1994) 62:3311-3319.

[90] Paradis S.E., Dubreuil J.D., Gottschalk M., Archambault M., Jacques M., Inhibition of adherence of Actinobacillus pleuropneumoniae to porcine respiratory tract cells by monoclonal antibodies directed against LPS and partial characterization of the LPS receptors, Curr. Microbiol. (1999) 39:313-320.

[91] Pol J.M.A., van Leengoed L.A.M.G., Stockhofe N., Kok G., Wensvoort G., Dual infections of PRRSV/ influenza or PRRSV/Actinobacillus pleuropneumoniae in the respiratory tract, Vet. Microbiol. (1997) 55:259 264.

[92] Provost M., Harel J., Labrie J., Sirois M., Jacques M., Identification, cloning and characterization of rfaE of Actinobacillus pleuropneumoniae serotype 1, a gene involved in lipopolysaccharide inner-core biosynthesis, FEMS Microbiol. Lett. (2003) $223: 7-14$

[93] Ramjeet M., Deslandes V., St Michael F., Cox A., Kobisch M.N., Gottschalk M., Jacques M., Truncation of the lipopolysaccharide outer core affects susceptibility to antimicrobial peptides and virulence of Actinobacillus pleuropneumoniae serotype 1, J. Biol. Chem. (2005) 280:39104-39114.

[94] Ramjeet M., Cox A.D., Hancock M.A., Mourez M., Labrie J., Gottschalk M., Jacques M., Mutation in the LPS outer core biosynthesis gene, galU, affects LPS interaction with the RTX toxins ApxI and ApxII and cytolytic activity of Actinobacillus pleuropneumoniae serotype 1, Mol. Microbiol. (2008) 70:221235 .

[95] Ramjeet M., Deslandes V., Gouré J., Jacques M., Actinobacillus pleuropneumoniae vaccines: from bacterins to new insights into vaccination strategies, Anim. Health Res. Rev. (2008) 9:25-45.

[96] Ricard M.A., Archibald F.S., Niven D.F., Isolation and identification of a putative porcine transferrin receptor from Actinobacillus pleuropneumoniae biotype 1, J. Gen. Microbiol. (1991) 137:2733-2740.

[97] Rioux S., Galarneau C., Harel J., Frey J., Nicolet J., Kobisch M., et al., Isolation and characterization of mini-Tn10 lipopolysaccharide mutants of Actinobacillus pleuropneumoniae serotype 1, Can. J. Microbiol. (1999) 45:1017-1026.

[98] Rioux S., Galarneau C., Harel J., Kobisch M., Frey J., Gottschalk M., Jacques M., Isolation and characterization of a capsule-deficient mutant of Actinobacillus pleuropneumoniae serotype 1, Microb. Pathog. (2000) 28:279-289.

[99] Rycroft A.N., Cullen J.M., Complement resistance in Actinobacillus (Haemophilus) 
pleuropneumoniae infection of swine, Am. J. Vet. Res. (1990) 51:1449-1453.

[100] Sakano T., Shibata I., Samegai Y., Taneda A., Okada M., Irisawa T., Sato S., Experimental pneumonia of pigs infected with Aujeszky's disease virus and Actinobacillus pleuropneumoniae, J. Vet. Med. Sci. (1993) 55:575-579.

[101] Schaller A., Kuhn R., Kuhnert P., Nicolet J., Anderson T.J., MacInnes J.I., et al., Characterization of apxIVA, a new RTX determinant of Actinobacillus pleuropneumoniae, Microbiology (1999) 145:21052116.

[102] Serebrin S., Rosendal S., Valdivieso-Garcia A., Little P., Endothelial cytotoxicity of Actinobacillus pleuropneumoniae, Res. Vet. Sci. (1991) 50:18-22.

[103] Shakarji L., Mikael L.G., Srikumar R., Kobisch M., Coulton J.W., Jacques M., FhuA and HgbA, outer membrane proteins of Actinobacillus pleuropneumoniae: their role as virulence determinants, Can. J. Microbiol. (2006) 52:391-396.

[104] Sheehan B.J., Langford P.R., Rycroft A.N., Kroll J.S., [Cu,Zn]-superoxide dismutase mutants of the swine pathogen Actinobacillus pleuropneumoniae are unattenuated in infections of the natural host, Infect. Immun. (2000) 68:4778-4781.

[105] Sheehan B.J., Bosse J.T., Beddek A.J., Rycroft A.N., Kroll J.S., Langford P.R., Identification of Actinobacillus pleuropneumoniae genes important for survival during infection in its natural host, Infect. Immun. (2003) 71:3960-3970.

[106] Sidibé M., Messier S., Lariviere S., Gottschalk M., Mittal K.R., Detection of Actinobacillus pleuropneumoniae in the porcine upper respiratory tract as a complement to serological tests, Can. J. Vet. Res. (1993) 57:204-208.

[107] Srikumar R., Mikael L.G., Pawelek P.D., Khamessan A., Gibbs B.F., Jacques M., Coulton J.W., Molecular cloning of haemoglobin-binding protein $\mathrm{HgbA}$ in the outer membrane of Actinobacillus pleuropneumoniae, Microbiology (2004) 150:17231734.

[108] Stevenson A., Macdonald J., Roberts M., Cloning and characterisation of type 4 fimbrial genes from Actinobacillus pleuropneumoniae, Vet. Microbiol. (2003) 92:121-134.

[109] Subashchandrabose S., LeVeque R.M., Wagner T.K., Kirkwood R.N., Kiupel M., Mulks M.H., Branched-chain amino acids are required for the survival and virulence of Actinobacillus pleuropneumoniae in swine, Infect. Immun. (2009) 77:49254933.
[110] Tarigan S., Slocombe R.F., Browning G.F., Kimpton W., Functional and structural changes of porcine alveolar macrophages induced by sublytic doses of a heat-labile, hemolytic, cytotoxic substance produced by Actinobacillus pleuropneumoniae, Am. J. Vet. Res. (1994) 55:1548-1557.

[111] Tegetmeyer H.E., Fricke K., Baltes N., An isogenic Actinobacillus pleuropneumoniae AasP mutant exhibits altered biofilm formation but retains virulence, Vet. Microbiol. (2009) 137:392-396.

[112] Tonpitak W., Thiede S., Oswald W., Baltes N., Gerlach G.F., Actinobacillus pleuropneumoniae iron transport: a set of $\operatorname{exb} B D$ genes is transcriptionally linked to the $t b p B$ gene and required for utilization of transferrin-bound iron, Infect. Immun. (2000) 68:1164-1170.

[113] Udeze F.A., Kadis S., Inhibition of bactericidal activity of anticapsular antibody by nonspecific antibodies reactive with surface-exposed antigenic determinants on Actinobacillus pleuropneumoniae, Infect. Immun. (1992) 60:3852-3860.

[114] Utrera V., Pijoan C., Fimbriae in Actinobacillus pleuropneumoniae strains isolated from pig respiratory tracts, Vet. Rec. (1991) 128:357-358.

[115] Van de Kerkhof A., Haesebrouck F., Chiers K., Ducatelle R., Kamp E.M., Smits M.A., Influence of Actinobacillus pleuropneumoniae and its metabolites on porcine alveolar epithelial cells, Infect. Immun. (1996) 64:3905-3907.

[116] Van Overbeke I., Chiers K., Charlier G., Vandenberghe I., Van Beeumen J., Ducatelle R., Haesebrouck F., Characterization of the in vitro adhesion of Actinobacillus pleuropneumoniae to swine alveolar epithelial cells, Vet. Microbiol. (2002) 88: 59-74.

[117] Vigre H., Angen O., Barfod K., Lavritsen D.T., Sorensen V., Transmission of Actinobacillus pleuropneumoniae in pigs under field-like conditions: emphasis on tonsillar colonisation and passively acquired colostral antibodies, Vet. Microbiol. (2002) 89:151-159.

[118] Ward C.K., Inzana T.J., Resistance of Actinobacillus pleuropneumoniae to bactericidal antibody and complement is mediated by capsular polysaccharide and blocking antibody specific for lipopolysaccharide, J. Immunol. (1994) 153:2110-2121.

[119] Ward C.K., Inzana T.J., Identification and characterization of a DNA region involved in the export of capsular polysaccharides by Actinobacillus pleuropneumoniae serotype 5a, Infect. Immun. (1997) 65:2491-2496. 
[120] Ward C.K., Lawrence M.L., Veit H.P., Inzana T.J., Cloning and mutagenesis of a serotype-specific DNA region involved in encapsulation and virulence of Actinobacillus pleuropneumoniae serotype 5a: concomitant expression of serotype $5 \mathrm{a}$ and 1 capsular polysaccharides in recombinant $A$. pleuropneumoniae serotype 1, Infect. Immun. (1998) 66:3326-3336.

[121] Wilke M., Franz B., Gerlach G.F., Characterization of a large transferrin-binding protein from Actinobacillus pleuropneumoniae serotype 7, J. Vet. Med. B Infect. Dis. Vet. Public Health (1997) 44:73-86.

[122] Wilson P.J., Falk G., Klashinsky S., Detection of Actinobacillus pleuropneumoniae infection in pigs, Can. Vet. J. (1987) 28:111-116.
[123] Yagihashi T., Nunoya T., Mitui T., Tajima M., Effect of Mycoplasma hyopneumoniae infection on the development of Haemophilus pleuropneumoniae in pigs, Nippon Juigaku Zasshi (1984) 46: 705-713.

[124] Zaas A.K., Schwartz D.A., Innate immunity and the lung: defense at the interface between host and environment, Trends Cardiovasc. Med. (2005) 15:195-202.

[125] Zhang Y., Tennent J.M., Ingham A., Beddome G., Prideaux C., Michalski W.P., Identification of type 4 fimbriae in Actinobacillus pleuropneumoniae, FEMS Microbiol. Lett. (2000) 189:15-18. 